\title{
Correction to: Phase I dose-finding study of eribulin and capecitabine for metastatic breast cancer: JBCRG-18 cape study
}

\author{
Masaya Hattori ${ }^{1} \cdot$ Hiroshi Ishiguro $^{2} \cdot$ Norikazu Masuda $^{3} \cdot$ Akiyo Yoshimura $^{1} \cdot$ Shoichiro Ohtani $^{4} \cdot$ Hiroyuki Yasojima $^{3}$. \\ Satoshi Morita ${ }^{5} \cdot$ Shinji Ohno ${ }^{6} \cdot$ Hiroji Iwata $^{1}$
}

Published online: 20 November 2017

(c) The Author(s) 2017. This article is an open access publication

\section{Correction to: Breast Cancer https://doi.org/10.1007/s12282-017-0798-4}

The article "Phase I dose-finding study of eribulin and capecitabine for metastatic breast cancer: JBCRG-18 cape study", written by Masaya Hattori, Hiroshi Ishiguro, Norikazu Masuda, Akiyo Yoshimura, Shoichiro Ohtani, Hiroyuki Yasojima, Satoshi Morita, Shinji Ohno and Hiroji Iwata, was originally published electronically on the publisher's Internet portal (currently SpringerLink) on 31 August 2017 without open access. With the author(s)' decision to opt for Open Choice the copyright of the article changed on 9 November 2017 to (C) The Author(s) 2017 and the article is forthwith distributed under the terms of the Creative Commons Attribution 4.0 International License (http://creativecommons. org/licenses/by/4.0/), which permits use, duplication, adaptation, distribution and reproduction in any medium or format, as long as you give appropriate credit to the original author(s) and the source, provide a link to the Creative Commons license and indicate if changes were made.

The original article was corrected.

Open Access This article is distributed under the terms of the Creative Commons Attribution 4.0 International License (http://creativecommons.org/licenses/by/4.0/), which permits unrestricted use, distribution, and reproduction in any medium, provided you give appropriate credit to the original author(s) and the source, provide a link to the Creative Commons license, and indicate if changes were made.

The original article can be found online at https://doi. org/10.1007/s12282-017-0798-4.

Hiroji Iwata

hiwata@aichi-cc.jp

1 Department of Breast Oncology, Aichi Cancer Center, 1-1

Kanokoden, Chikusa-ku, Nagoya 464-8681, Japan

2 Department of Target Therapy Oncology, Graduate School of Medicine, Kyoto University, Kyoto 606-8501, Japan

3 Department of Surgery, Breast Oncology, NHO Osaka National Hospital, Osaka 540-0006, Japan

4 Division of Breast Surgery, Hiroshima City Hiroshima Citizens Hospital, Hiroshima 730-8518, Japan

5 Department of Biomedical Statistics and Bioinformatics, Graduate School of Medicine, Kyoto University, Kyoto 606-8501, Japan

6 Breast Oncology Center, Cancer Institute Hospital of JFCR, Tokyo 135-8550, Japan 